\title{
Jahn-Teller Distortion in Copper(II) Complexes. Why Tetragonal Elongation is Preferred to Tetragonal Compression
}

\author{
HIDEO YAMATERA
}

Department of Chemistry, Faculty of Science, Nagoya University, Chikusa-ku, Nagoya 464, Japan*

Dedicated to Jannik Bjerrum on the occasion of his 70 th birthday

The tetragonal elongation of octahedral copper(II) complexes is interpreted by considering interactions with excited states. The stabilization due to interactions with the lowest excited configurations of the copper(II) ion, with an electron excited to the $4 s$ orbital or to the vacancy in the $3 d$ shell, are estimated to be greater for the lowest ${ }^{2} B_{1 g}$ state of an elongated octahedron than for the lowest ${ }^{2} A_{1 g}$ state of a compressed octahedron. Discussions are made on the bases of the electrostatic and molecularorbital theories.

Six-coordinate copper(II) complexes have long been known to assume distorted octahedral shapes, in accordance with the theoretical prediction of Jahn and Teller. ${ }^{1}$ Experimentally, most of the copper(II) complexes were found to be elongated along the tetragonal axis. Many copper(II) complexes of orthorhombic symmetry can also be approximated by tetragonally elongated octahedra. ${ }^{2}$ The only exception would be $\mathrm{K}_{2} \mathrm{CuF}_{4}$, in which tetragonally compressed octahedra were observed. ${ }^{3}$ However, an X-ray re-examination recently performed by Haegele and $\mathrm{Babel}^{4}$ revealed the existence of a superstructure and elongated $\mathrm{CuF}_{6}$ octahedra. Friebel and Reinen, ${ }^{5}$ on the ground of their ESR study, concluded that in no copper(II) compounds copper(II) ions have been observed in tetragonally compressed octahedra of six identical ligands. In a very recent investigation of the crystal structure of

\footnotetext{
* Part of this work was done at Department I, Inorganic Chemistry, H. C. Ørsted Institute, University of Copenhagen (July - September, 1977) on leave from Nagoya University.
}

bis(terpyridine)copper(II) nitrate, Allmann et al. ${ }^{6}$ have gathered strong evidence against the tetragonal compression of octahedral copper(II) complexes; the compressed octahedron, which would be formed by the coordination of two terpyridine molecules with the minimum distortion in the ligand structure, actually undergoes a rhombic distortion to approach a tetragonally elongated octahedron.

Although theoretical interpretations for the tetragonal elongation have already been given by Öpik and Pryce ${ }^{7}$ and also by Liehr and Ballhausen, ${ }^{8}$ they do not seem sufficient to explain this strong tendency to tetragonal elongation. The present paper provides an additional basis for such a feature characteristic of the Jahn-Teller distortion of octahedral copper(II) complexes. The discussion is first made for the sake of simplicity from the electrostatic point of view and then on the basis of the molecularorbital theory.

\section{ELECTROSTATIC CONSIDERATION}

The ${ }^{2} E_{g}$ ground state of the regular-octahedral copper(II) complex is known to be Jahn-Teller unstable and to be deformed to give the ${ }^{2} A_{1 g}$ or ${ }^{2} B_{1 g}$ ground state; the energies of these two states are to a first approximation identical. ${ }^{7,8}$ However, each of these states is subjected to configuration interaction, or mixed with excited states, to result in an energy decrease. The lowest excited states are those containing the copper(II) ion of the $3 d^{8} 4 s$ configuration. If the complex takes the ${ }^{2} A_{1 g}$ ground state, one of the wave functions of the ground doublet can be expressed by: 


$$
\Psi_{0 A}=\left|(d \pi s)^{2}(d \pi c)^{2}(d \delta s)^{2}(d \delta c)^{2}(d \sigma)^{+}\right|
$$

where $(d \sigma)^{+}$represents a positive-spin electron occupying the $d \sigma$ orbital and where $(d \pi s)^{2}$, for example, stands for $(d \pi s)^{+}(d \pi s)^{-}$. (For the notations of orbitals, refer to Schäffer and Jørgensen. ${ }^{9}$ ) In this expression, the orbitals of lower energies and the normalization constant are omitted for the sake of simplicity. The only ${ }^{2} A_{1 g}$ excited state resulting from the excitation of a $3 d$ electron to the $4 s$ orbital without spin change is:

$$
\Psi_{1 A}=\left|(d \pi s)^{2}(d \pi c)^{2}(d \delta s)^{2}(d \delta c)^{2}(s)^{+}\right|
$$

On the other hand, if the complex takes the ${ }^{2} B_{1 g}$ ground state, the ground and excited states with $+\frac{1}{2}$ spin are:

$$
\begin{aligned}
\Psi_{0 B}= & \left|(d \pi s)^{2}(d \pi c)^{2}(d \delta s)^{2}(d \sigma)^{2}(d \delta c)^{+}\right| \\
\Psi_{1 B}= & (1 / \sqrt{2})\left[\left|(d \pi s)^{2}(d \pi c)^{2}(d \delta s)^{2}(d \sigma)^{+}(s)^{-}(d \delta c)^{+}\right|\right. \\
& \left.+\left|(d \pi s)^{2}(d \pi c)^{2}(d \delta s)^{2}(s)^{+}(d \sigma)^{-}(d \delta c)^{+}\right|\right]
\end{aligned}
$$

The magnitude of configuration interaction is approximately equal to the square of the nondiagonal element of the energy matrix divided by the energy difference between the interacting states. The nondiagonal elements between the ground and excited states of the ${ }^{2} A_{1 g}$ and ${ }^{2} B_{1 g}$ symmetries are:

$$
\begin{aligned}
& \left\langle\Psi_{0 A}\left|H_{\mathrm{c}}\right| \Psi_{1 A}\right\rangle=\left\langle d \sigma\left|U_{\mathrm{c}}\right| s\right\rangle+\text { (two-electron terms) } \\
& \left\langle\Psi_{0 B}\left|H_{\mathrm{e}}\right| \Psi_{1 B}\right\rangle=\sqrt{2}\left\langle d \sigma\left|U_{\mathrm{e}}\right| s\right\rangle+\text { (two-electron terms) }
\end{aligned}
$$

where $U_{\mathrm{c}}$ and $U_{\mathrm{e}}$ represent the one-electron part of $H_{\mathrm{c}}$ and $H_{\mathrm{e}}$, the Hamiltonian operators for the compressed and elongated complexes, respectively. Since the energy difference between $\Psi_{1 A}$ and $\Psi_{0 A}$ is in the zeroth order the same as that between $\Psi_{1 B}$ and $\Psi_{O B}$, the configuration interaction in the ${ }^{2} B_{1 g}$ case will be about twice as great as that in the ${ }^{2} A_{1 g}$ case, provided that the two-electron terms are small enough or roughly proportional to the one-electron terms. Interaction with the excited states that have two electrons excited to the orbitals (including the spin function) not occupied in the ground state was also ignored, since the nondiagonal matrix elements between these excited states and the ground state contain no one-electron terms and thus will be small enough to be ignored.

The excitation of a $3 d$ electron to a $4 d$ or a $5 s$ orbital can also result in excited states of the same symmetry as of the ground state $\left({ }^{2} A_{1 g}\right.$ or $\left.{ }^{2} B_{1 g}\right)$.
These excited states will interact with the ground state to lead a stabilization of the latter, but to a much smaller extent than the cases discussed above and without any preferential stabilization of the ${ }^{2} A_{1 g}$ ground state.

The stabilization of the ${ }^{2} B_{1 g}$ ground state through configuration interaction is therefore nearly twice as great as that of the lowest ${ }^{2} A_{1 g}$ state, if effects of secondary importance are ignored. This can be the explanation of why the tetragonal elongation is preferred to the tetragonal compression in octahedral copper(II) complexes, on the assumption of the electrostatic model of the complex.

\section{MOLECULAR-ORBITAL CONSIDERATION}

In the molecular-orbital approach, on has to take into account configuration interactions with charge-transfer excited states in addition to those with the states arisen from the excitation within the copper(II) ion. For the reason described above in the electrostatic consideration, only those excited states that will result from one-electron excitation will be considered.

Table 1. Zeroth-order wave functions of the ground and excited states of the ${ }^{2} A_{1 g}$ and ${ }^{2} A B_{1 g}$ symmetries. ${ }^{a}$

$$
\begin{aligned}
\Psi_{0 A}^{\circ}= & \left|\cdots(\phi s)^{2}(\phi \delta c)^{2}(\phi \sigma)^{2}(d \delta c)^{2}(d \sigma)^{+}\right| \\
\Psi_{1 A}^{\circ}= & \cdots(\phi s)^{2}(\phi \delta c)^{2}(\phi \sigma)^{2}(d \delta c)^{2}(s)^{+} \mid \\
\Psi_{2 A}^{\circ}= & \cdots(\phi s)^{+}(d \sigma)^{-}(\phi \delta c)^{2}(\phi \sigma)^{2}(d \delta c)^{2}(d \sigma)^{+} \mid \\
\Psi_{3 A}^{\circ}= & \left|\cdots(\phi s)^{2}(\phi \delta c)^{2}(\phi \sigma)^{+}(d \sigma)^{-}(d \delta c)^{2}(d \sigma)^{+}\right| \\
\Psi_{4 A}^{\circ}= & (1 / \sqrt{2})\left[\left|\cdots(\phi s)^{+}(s)^{-}(\phi \delta c)^{2}(\phi \sigma)^{2}(d \delta c)^{2}(d \sigma)^{+}\right|\right. \\
& \left.+\left|\cdots(s)^{+}(\phi s)^{-}(\phi \delta c)^{2}(\phi \sigma)^{2}(d \delta c)^{2}(d \sigma)^{+}\right|\right] \\
\Psi_{5 A}^{\circ}= & (1 / \sqrt{2})\left[\left|\cdots(\phi s)^{2}(\phi \delta c)^{2}(\phi \sigma)^{+}(s)^{-}(d \delta c)^{2}(d \sigma)^{+}\right|\right. \\
& \left.+\left|\cdots(\phi s)^{2}(\phi \delta c)^{2}(s)^{+}(\phi \sigma)^{-}(d \delta c)^{2}(d \sigma)^{+}\right|\right] \\
\Psi_{0 B}^{\circ}= & \left|\cdots(\phi s)^{2}(\phi \delta c)^{2}(\phi \sigma)^{2}(d \sigma)^{2}(d \delta c)^{+}\right| \\
\Psi_{1 B}^{\circ}= & (1 / \sqrt{2})\left[\left|\cdots(\phi s)^{2}(\phi \delta c)^{2}(\phi \sigma)^{2}(d \sigma)^{+}(s)^{-}(d \delta c)^{+}\right|\right. \\
& \left.+\left|\cdots(\phi s)^{2}(\phi \delta c)^{2}(\phi \sigma)^{2}(s)^{+}(d \sigma)^{-}(d \delta c)^{+}\right|\right] \\
\Psi_{2 B}^{\circ}= & \left|\cdots(\phi s)^{2}(\phi \delta c)^{+}(d \delta c)^{-}(\phi \sigma)^{2}(d \sigma)^{2}(d \delta c)^{+}\right| \\
\Psi_{3 B}^{\circ}= & (1 / \sqrt{2})\left[\left|\cdots(\phi s)^{+}(s)^{-}(\phi \delta c)^{2}(\phi \sigma)^{2}(d \sigma)^{2}(d \delta c)^{+}\right|\right. \\
& \left.+\left|\cdots(s)^{+}(\phi s)^{-}(\phi \delta c)^{2}(\phi \sigma)^{2}(d \sigma)^{2}(d \delta c)^{+}\right|\right] \\
\Psi_{4 B}^{\circ}= & (1 / \sqrt{2})\left[\left|\cdots(\phi s)^{2}(\phi \delta c)^{2}(\phi \sigma)^{+}(s)^{-}(d \sigma)^{2}(d \delta c)^{+}\right|\right. \\
& \left.+\left|\cdots(\phi s)^{2}(\phi \delta c)^{2}(s)^{+}(\phi \sigma)^{-}(d \sigma)^{2}(d \delta c)^{+}\right|\right]
\end{aligned}
$$

${ }^{a}$ The $\Psi_{0 A}^{\circ}$ or $\Psi_{0 B}^{\circ}$ state will be the ground state according to whether the complex is tetragonally compressed or elongated. 
Noting in particular the $d \delta c, d \sigma$, and $s$ orbitals together with the corresponding ligand orbitals, $\phi \delta c, \phi \sigma$, and $\phi s$, and omitting other orbitals, one can describe the ground and excited states as listed in Table 1.

The orbitals, $\phi s, d \sigma$, and $s$, for example, are not pure ligand and metal orbitals, but are molecular orbitals expressed by:

$$
\begin{array}{ll}
(\phi s)=a_{1}(\mathrm{~L} s)+b_{1}(\mathrm{M} s) & \left(a_{1}>b_{1}>0\right) \\
(d \sigma)=a_{2}(\mathrm{M} d \sigma)-b_{2}(\mathrm{~L} d \sigma) & \left(a_{2}>b_{2}>0\right) \\
(s)=a_{3}(\mathrm{M} s)-b_{3}(\mathrm{~L} s) & \left(a_{3}>b_{3}>0\right)
\end{array}
$$

where $(\mathrm{M} s)$ and $(\mathrm{M} d \sigma)$ represent the pure $4 s$ and $3 d \sigma$ orbitals of the copper(II) ion and where (Ls) and $(L d \sigma)$ are the molecular orbitals consisting exclusively of the ligand $\sigma$ orbitals and having the symmetries corresponding to $s$ and $d \sigma$, respectively. Thus they are expressed by:

$$
\begin{aligned}
& (\mathrm{Ls})=(1 / \sqrt{6})[(z)+(\bar{z})+(x)+(\bar{x})+(y)+(\bar{y})] \\
& (\mathrm{L} d \sigma)=(1 / \sqrt{12})[2(z)+2(\bar{z})-(x)-(\bar{x})-(y)-(\bar{y})]
\end{aligned}
$$

where $(z)$ and $(\bar{z})$, for example, are the $\sigma$ orbitals of the ligands lying on the $z$ and $-z$ axes, respectively.

Considering configuration interactions with the excited states listed in Table 1 , the energies of the lowest ${ }^{2} A_{1 g}$ and ${ }^{2} B_{1 g}$ states can be given by:

$$
\begin{aligned}
& E_{0 A}=E_{0 A}^{\circ}-\sum_{n=1}^{5}\left[\left.\left\langle\Psi_{n A}^{\circ}\left|H_{\mathrm{c}}\right| \Psi_{0 A}^{\circ}\right\rangle\right|^{2} /\left(E_{n A}^{\circ}-E_{0 A}^{\circ}\right)\right] \\
& E_{0 B}=E_{0 B}^{\circ}-\sum_{n=1}^{4}\left[\left|\left\langle\Psi_{n B}^{\circ}\left|H_{\mathrm{e}}\right| \Psi_{0 B}^{\circ}\right\rangle\right|^{2} /\left(E_{n B}^{\circ}-E_{0 B}^{\circ}\right)\right]
\end{aligned}
$$

where $E_{n A}^{\circ}$ and $E_{n B}^{\circ}$ (including $n=0$ ) are the energies of $\Psi_{n A}^{\circ}$ and $\Psi_{n B}^{\circ}$, and where $H_{\mathrm{c}}$ and $H_{\mathrm{e}}$ represent the Hamiltonian operators for the tetragonally compressed and elongated complexes, respectively. The degree of distortion is assumed to be the same for the compressed and elongated complexes.

Referring to the wave functions given in Table 1 and ignoring two-electron integrals, one can simplify each matrix element appearing in eqns. (4) and (5) into the form of a single one-electron integral. The results are shown in Table 2.

On the assumptions and approximations adopted here, one has the equations: $E_{0 A}^{\circ}=E_{0 B}^{\circ}, E_{1 A}^{\circ}=E_{1 B}^{\circ}$, $E_{3 A}^{\circ}=E_{2 B}^{\circ}, \quad E_{4 A}^{\circ}=E_{3 B}^{\circ}, \quad E_{5 A}^{\circ}=E_{4 B}^{\circ}, \quad\left\langle d \sigma\left|H_{\mathrm{c}}\right| \phi \sigma\right\rangle=$ $\left\langle d \delta c\left|H_{\mathrm{e}}\right| d \delta c\right\rangle$, and $\left\langle\kappa\left|H_{\mathrm{c}}\right| \lambda\right\rangle\left|=\left\langle\kappa\left|H_{\mathrm{e}}\right| \lambda\right\rangle\right|$ for all other one-electron integrals in Table 2 . Then comparisons of the matrix elements related to $\Psi_{0 A}^{\circ}$ with those related to $\Psi_{0 B}^{\circ}$ in Table 2 show that the energy difference between the lowest ${ }^{2} A_{1 g}$ and ${ }^{2} B_{1 g}$ states can be given by:

$$
\begin{aligned}
E_{0 A}-E_{0 B}= & -\left.\left\langle d \sigma\left|H_{\mathrm{c}}\right| \phi s\right\rangle\right|^{2} /\left(E_{2 A}^{\circ}-E_{0 A}^{\circ}\right) \\
& +\left.\left\langle s\left|H_{\mathrm{e}}\right| d \sigma\right\rangle\right|^{2} /\left(E_{1 B}^{\circ}-E_{0 B}^{\circ}\right)
\end{aligned}
$$

Using eqns. (1)-(3), the matrix elements appearing in eqn. (6) can be expressed as:

$$
\begin{gathered}
\left\langle d \sigma\left|H_{\mathrm{c}}\right| \phi s\right\rangle=\left\langle\phi s\left|H_{\mathrm{c}}\right| d \sigma\right\rangle \\
=a_{1} a_{2}\left\langle\mathrm{~L} s\left|H_{\mathrm{c}}\right| \mathrm{M} d \sigma\right\rangle-a_{1} b_{2}\left\langle\mathrm{Ls}\left|H_{\mathrm{c}}\right| \mathrm{L} d \sigma\right\rangle \\
+b_{1} a_{2}\left\langle\mathrm{M} s\left|H_{\mathrm{c}}\right| \mathrm{M} d \sigma\right\rangle-b_{1} b_{2}\left\langle\mathrm{M} s\left|\mathrm{H}_{\mathrm{c}}\right| \mathrm{L} d \sigma\right\rangle(7) \\
\left\langle s\left|H_{\mathrm{e}}\right| d \sigma\right\rangle=a_{2} a_{3}\left\langle\mathrm{M} s\left|H_{\mathrm{e}}\right| \mathrm{M} d \sigma\right\rangle-b_{2} a_{3}\left\langle\mathrm{M} s\left|H_{\mathrm{e}}\right| \mathrm{L} d \sigma\right\rangle \\
-a_{2} b_{3}\left\langle\mathrm{~L} s\left|H_{\mathrm{e}}\right| \mathrm{M} d \sigma\right\rangle+b_{2} b_{3}\left\langle\mathrm{~L} s\left|H_{\mathrm{e}}\right| \mathrm{L} d \sigma\right\rangle \quad(8)
\end{gathered}
$$

If the complex is of regular octahedron, all the four matrix elements appearing on the right-hand side of each equation vanish from the symmetry requirement. When the octahedron is elongated along the $z$ axis and compressed along the $x$ and $y$ axes, the absolute magnitude of the energy of interaction between an orbital of the metal and a $\sigma$ orbital of a ligand will decrease for the ligands on the $z$ axis and increase for the ligands on the $x$ and $y$ axes. Since the interaction energy is negative for a positive overlap and positive for a negative overlap of the orbital

Table 2. Reduction of the matrix elements between states into one-electron integrals (two-electron integrals were ignored because of their secondary importance).

$$
\begin{aligned}
\left\langle\Psi_{1 A}^{\circ}\left|H_{\mathrm{c}}\right| \Psi_{0 A}^{\circ}\right\rangle & =\left\langle s\left|H_{\mathrm{c}}\right| d \sigma\right\rangle \\
\left\langle\Psi_{2 A}^{\circ}\left|H_{\mathrm{c}}\right| \Psi_{0 A}^{\circ}\right\rangle & =\left\langle d \sigma\left|H_{\mathrm{c}}\right| \phi s\right\rangle \\
\left\langle\Psi_{3 A}^{\circ}\left|H_{\mathrm{c}}\right| \Psi_{0 A}^{\circ}\right\rangle & =\left\langle d \sigma\left|H_{\mathrm{c}}\right| \phi \sigma\right\rangle \\
\left\langle\Psi_{4 A}^{\circ}\left|H_{\mathrm{c}}\right| \Psi_{0 A}^{\circ}\right\rangle & =\sqrt{2}\left\langle s\left|H_{\mathrm{c}}\right| \phi s\right\rangle \\
\left\langle\Psi_{5 A}^{\circ}\left|H_{\mathrm{c}}\right| \Psi_{0 A}^{\circ}\right\rangle & =\sqrt{2}\left\langle s\left|H_{\mathrm{c}}\right| \phi \sigma\right\rangle
\end{aligned}
$$

$$
\begin{aligned}
& \left\langle\Psi_{1 B}^{\circ}\left|H_{\mathrm{e}}\right| \Psi_{0 B}^{\circ}\right\rangle=\sqrt{2}\left\langle s\left|H_{\mathrm{e}}\right| d \sigma\right\rangle \\
& - \\
& \left\langle\Psi_{2 B}^{\circ}\left|H_{\mathrm{e}}\right| \Psi_{0 B}^{\circ}\right\rangle=\left\langle d \delta c\left|H_{\mathrm{e}}\right| \phi \delta c\right\rangle \\
& \left\langle\Psi_{3 B}^{\circ}\left|H_{\mathrm{e}}\right| \Psi_{0 B}^{\circ}\right\rangle=\sqrt{2}\left\langle s\left|H_{\mathrm{e}}\right| \phi s\right\rangle \\
& \left\langle\Psi_{4 B}^{\circ}\left|H_{\mathrm{e}}\right| \Psi_{0 B}^{\circ}\right\rangle=\sqrt{2}\left\langle s\left|H_{\mathrm{e}}\right| \phi \sigma\right\rangle
\end{aligned}
$$


lobes, both $\left\langle\mathrm{Ls}\left|H_{\mathrm{e}}\right| \mathrm{M} d \sigma\right\rangle$ and $\left\langle\mathrm{Ms}\left|H_{\mathrm{e}}\right| \mathrm{L} d \sigma\right\rangle$ will have positive values for a tetragonally elongated octahedron. The situation with a tetragonally compressed octahedron is analogous to that described above except that the signs of energy changes are inversed. Thus $\left\langle\mathrm{L} s\left|H_{\mathrm{c}}\right| \mathrm{M} d \sigma\right\rangle$ and $\left\langle\mathrm{M} s\left|H_{\mathrm{c}}\right| \mathrm{L} d \sigma\right\rangle$ will be positive for a tetragonally compressed octahedron.

The tetragonal distortion also affects the electrostatic energy. The electrostatic repulsive effect of the ligand on a metal electron will be decreased in the $z$ direction but increased in the $x$ and $y$ directions by the tetragonal elongation of the complex. This leads to a negative value of $\left\langle\mathrm{Ms}\left|H_{\mathrm{e}}\right| \mathrm{M} d \sigma\right\rangle$ with due consideration for the signs of the $\mathbf{M} d \sigma$ orbital lobes. The electrostatic effect of the metal ion on a ligand electron is attractive, and therefore $\left\langle\mathrm{L} s\left|H_{\mathrm{e}}\right| \mathrm{L} d \sigma\right\rangle$ will be positive. Tetragonal compression, on the other hand, will result in positive $\left\langle\mathrm{Ms}\left|H_{\mathrm{c}}\right| \mathrm{M} d \sigma\right\rangle$ and negative $\left\langle\mathrm{Ls}\left|H_{\mathrm{c}}\right| \mathrm{L} d \sigma\right\rangle$ values.

Now we shall compare the energy of the ground state of a tetragonally compressed complex $\left(E_{0 A}\right)$ with that for a tetragonally elongated complex $\left(E_{0 B}\right)$, referring to eqns. (6) $-(8)$. We shall first consider the case of an essentially ionic complex. In this case, the $b_{i}$ coefficients $(i=1-3)$ are negligibly small, so that eqns. (7) and (8) become:

$$
\begin{aligned}
& \left\langle d \sigma\left|H_{\mathrm{c}}\right| \phi s\right\rangle \approx\left\langle\mathrm{L} s\left|H_{\mathrm{c}}\right| \mathrm{M} d \sigma\right\rangle \\
& \left\langle s\left|H_{\mathrm{e}}\right| d \sigma\right\rangle \approx\left\langle\mathrm{M} s\left|H_{\mathrm{e}}\right| \mathrm{M} d \sigma\right\rangle
\end{aligned}
$$

The $\left\langle\mathrm{L} s\left|H_{\mathrm{c}}\right| \mathrm{M} d \sigma\right\rangle$ matrix element represents a covalent interaction and will be very small for an essentially ionic complex.

Hence:

$$
\left|\left\langle d \sigma\left|H_{\mathrm{c}}\right| \phi s\right\rangle\right| \ll\left|\left\langle s\left|H_{\mathrm{e}}\right| d \sigma\right\rangle\right|
$$

The eqn. (6) shows that the ${ }^{2} B_{1 g}$ ground state is more stable than the ${ }^{2} A_{1 g}$ ground state, or a tetragonal elongation results, in agreement with the prediction from the electrostatic consideration.

When the coordinate bonds are of highly covalent character, the matrix elements representing covalent interactions are of prime importance in eqns. (7) and (8) and those representing ionic interactions can be ignored. In eqn. (7), the terms containing $\left\langle\mathrm{L} s\left|H_{\mathrm{c}}\right| \mathrm{M} d \sigma\right\rangle$ and $\left\langle\mathrm{M} s\left|H_{\mathrm{c}}\right| \mathrm{L} d \sigma\right\rangle$ are partially cancelled out to result in a smaller balance for the value of $\left\langle d \sigma\left|H_{c}\right| \phi s\right\rangle$, whereas in eqn. (8), the covalent terms are added together to give a larger value for $\left\langle s\left|H_{\mathrm{e}}\right| d \sigma\right\rangle$. In this connection, it should be noted that the values of $b_{i}$ are not very different from those of $a_{i}$ for an essentially covalent bonding. An example of calculation is given below to make the situation clear. If we assume that the overlap integrals between the metal and ligand-group orbitals are equal to 0.3 and that each electronic charge is distributed between the metal and the ligands in the ratio of $1: 3$ for the $\phi s$ orbital and $3: 1$ for the $d \sigma$ and $s$ orbitals, then the calculation gives 0.81 and 0.39 for the former orbital and 0.97 and 0.66 for the latter orbitals as the values of the $a_{i}$ and $b_{i}$ coefficients, respectively. These $a_{i}$ and $b_{i}$ values will lead to a ratio of $\left|\left\langle s\left|H_{\mathrm{e}}\right| d \sigma\right\rangle\right| /\left|\left\langle d \sigma\left|H_{\mathrm{c}}\right| \phi s\right\rangle\right|$ greater than two, with the assumption that the matrix elements under consideration (those representing covalent interactions) are equal in their absolute values. An examination in more detail shows that $\left\langle\mathrm{M} s\left|H_{\mathrm{c}}\right| \mathrm{L} d \sigma\right\rangle \mid$ is probably greater than $\left|\left\langle\mathrm{L} s\left|H_{\mathrm{c}}\right| \mathrm{M} d \sigma\right\rangle\right|$, since the $\sigma$ orbital of each ligand will overlap more favourably with the farther-reaching $4 s$ orbital than with the $3 d$ orbital. This consideration will make the estimated $\left|\left\langle s\left|H_{\mathrm{e}}\right| d \sigma\right\rangle\right| /\left|\left\langle d \sigma\left|H_{\mathrm{c}}\right| \phi s\right\rangle\right|$ ratio even greater than given above.

A similar result can also be obtained in an intermediate or weakly covalent case. In this case, all the four matrix elements appearing on the right-hand sides of eqn. (7) and (8) will be of comparable magnitudes, but have coefficients of somewhat different magnitudes. The coefficient of the first term is the greatest and that of the last term is the smallest in each equation. In eqn. (7) for a compressed octahedron, the first term is negative and the other terms are positive, whereas the three important terms of eqn. (8) are all negative and the least important term (the last term) is positive for an elongated octahedron. Although a definite prediction awaits the quantitative evaluation of the matrix elements, it can be expected that the relation, $\left|\left\langle s\left|H_{\mathrm{e}}\right| d \sigma\right\rangle\right| /\left|\left\langle d \sigma\left|H_{\mathrm{c}}\right| \phi s\right\rangle\right|>1$, is hold in the intermediate cases. The example of calculation shown above in connection with the essentially covalent case is actually that for a case of $50 \%$ covalent character. The $\left|\left\langle s\left|H_{\mathrm{e}}\right| d \sigma\right\rangle\right| /\left|\left\langle d \sigma\left|H_{\mathrm{c}}\right| \phi s\right\rangle\right|$ ratio obtained above is actually a lower limit and the ratio would further be increased by the consideration of ionic terms. This example would suggest that there would probably be a smooth change in the $\left|\left\langle s\left|H_{\mathrm{e}}\right| d \sigma\right\rangle\right| /\left\langle d \sigma\left|H_{\mathrm{c}}\right| \phi s\right\rangle \mid$ ratio in going from a covalent complex to an ionic complex and that the ratio would always be considerably greater than unity. 
Now it has been shown that $\left|\left\langle s\left|H_{\mathrm{e}}\right| d \sigma\right\rangle\right|^{2}$ can be expected to be much greater than $\left|\left\langle d \sigma\left|H_{\mathrm{c}}\right| \phi s\right\rangle\right|^{2}$. The dominators of the terms on the right-hand side of eqn. (6) are of the same order of magnitude and are not likely to differ from each other by a large factor (say a factor exceeding two or three). Then the right-hand side of eqn. (6) gives a positive value definitely in the extreme cases and very probably in the intermediate cases. This leads to the result that the $\Psi_{O B}$ state for a tetragonally elongated complex is more stable than the $\Psi_{0 A}$ state for a compressed complex. In the author's opinion, the greater stabilization due to configuration interaction of the ground state, $\Psi_{0 B}$, of the tetragonally elongated octahedron is the main or at least an important reason for the tetragonal elongation observed in octahedral copper(II) complexes.

Acknowledgements. The author would like to thank Professor Jannik Bjerrum for offering him the opportunity of studying at his laboratory. The author is grateful to Dr. Claus Schäffer for the stimulating discussions without which this work would not have been completed. The author also thanks the Ministry of Education of the Government of Japan for leave and the financial assistance which enabled him to study at the University of Copenhagen.

\section{REFERENCES}

1. Jahn, H. A. and Teller, E. Proc. R. Soc. London Ser. A 161 (1937) 220.

2. Gazo, J., Bersuker, I. B., Garaj, J., Kabesová, M., Kohout, J., Langfelderová, H., Melník, M., Serátor, M. and Valach, F. Coord. Chem. Rev. 19 (1976) 253.

3. Know, K. J. Chem. Phys. 30 (1959) 991.

4. Babel, D. Struct. Bonding (Berlin) 3 (1967) 36; Haegele, R. and Babel, D. Z. Anorg. Allg. Chem. 409 (1974) 11.

5. Friebel, C. and Reinen, D. Z. Anorg. Allg. Chem. 407 (1974) 193.

6. Allmann, R., Henke, W. and Reinen, D. Inorg. Chem. 17 (1978) 378.

7. Öpik, U. and Pryce, M. H. L. Proc. R. Soc. London Ser. A 238 (1957) 425.

8. Liehr, A. D. and Ballhausen, C. J. Ann. Phys. 3 (1958) 304.

9. Schäffer, C. E. Struct. Bonding (Berlin) 5 (1968) 68; Pure Appl. Chem. 24 (1970) 366; Jørgensen, C. K. Modern Aspects of Ligand Field Theory, North-Holland, Amsterdam 1971, p. 19.

Received September 25, 1978.

Acta Chem. Scand. A 33 (1979) No. 2 